\title{
Freiberg's Infraction of the Second Metatarsal Head with Bioorthologic Repair using the NEXA Osteocurerм Bone Graft: A case report
}

\author{
by Al Kline, $\mathrm{DPM}^{1}$
}

The Foot \& Ankle Journal 1 (11): 4

Freiberg's infraction is a relatively common osteochondritis of the second metatarsal head. It appears to affect young women who are active in sports. Its etiology is thought to be traumatic in nature causing a painful alteration, subchondral disruption and collapse of the articular cartilage. A case report is presented describing this disorder in a young female runner. The use of a bioorthologic bone plug to surgically treat this condition is discussed and presented. It appears that the use of bioorthologic materials provide a promising alternative to surgical osteotomies. It has been shown that bioorthologic materials will provide a porous scaffold allowing blood, marrow and progenitor cells to wick into the pores and provide a stable environment for tissue in-growth and cellular development. Between six and twelve months, the polymer is resorbed and replaces the natural hyaline cartilage of the joint surface.

Key words: Freiberg's infraction, osteochondritis, osteochondritis dessicans, bioorthologic bone graft

Accepted: October , 2008

Published: November, 2008

This is an Open Access article distributed under the terms of the Creative Commons Attribution License. It permits unrestricted use, distribution, and reproduction in any medium, provided the original work is properly cited. OThe Foot \& Ankle Journal (www.faoj.org)

In 1914, Alfred H. Freiberg was the first to describe a painful collapse to the articular surface of the second metatarsal head. ${ }^{1}$ The etiology of injury can be attributed to trauma and subchondral disruption of the vascular supply to the articular cartilage. The incidence of injury appears to be most commonly found in young women and girls, although the overall incidence of osteochondroses is higher in men. ${ }^{2}$ On occasion, the third metatarsal head may also be involved. Osteochondritis, in effect, can involve any of the five metatarsal heads of the foot. However, the incidence is highest involving the second metatarsal head. ${ }^{1}$

Address correspondence to: Al Kline, DPM

3130 South Alameda, Corpus Christi, Texas 78404.

${ }^{1}$ Adjunct Clinical Faculty, Barry University School of Podiatric Medicine. Private practice, Chief of Podiatry, Doctors Regional Medical Center. Corpus Christi, Texas, 78411.
There does not appear to be a genetic link to Freiberg's infraction, although a recent report of Freiberg's infraction in twins may suggest an underlying genetic predisposition. ${ }^{8}$

In Freiberg's original article, he described six women with a painful limp and pain isolated to the second metatarsal head. Radiographs revealed collapse of the articular surface of the second metatarsal head with intra-articular loose bodies seen in three of the six patients. He noted that four of the six patients were under 18 years of age and postulated that a long second metatarsal combined with an ineffective first ray complex attributed to overload of the second metatarsal phalangeal (MTP) joint and subsequent articular collapse. $^{2}$ 


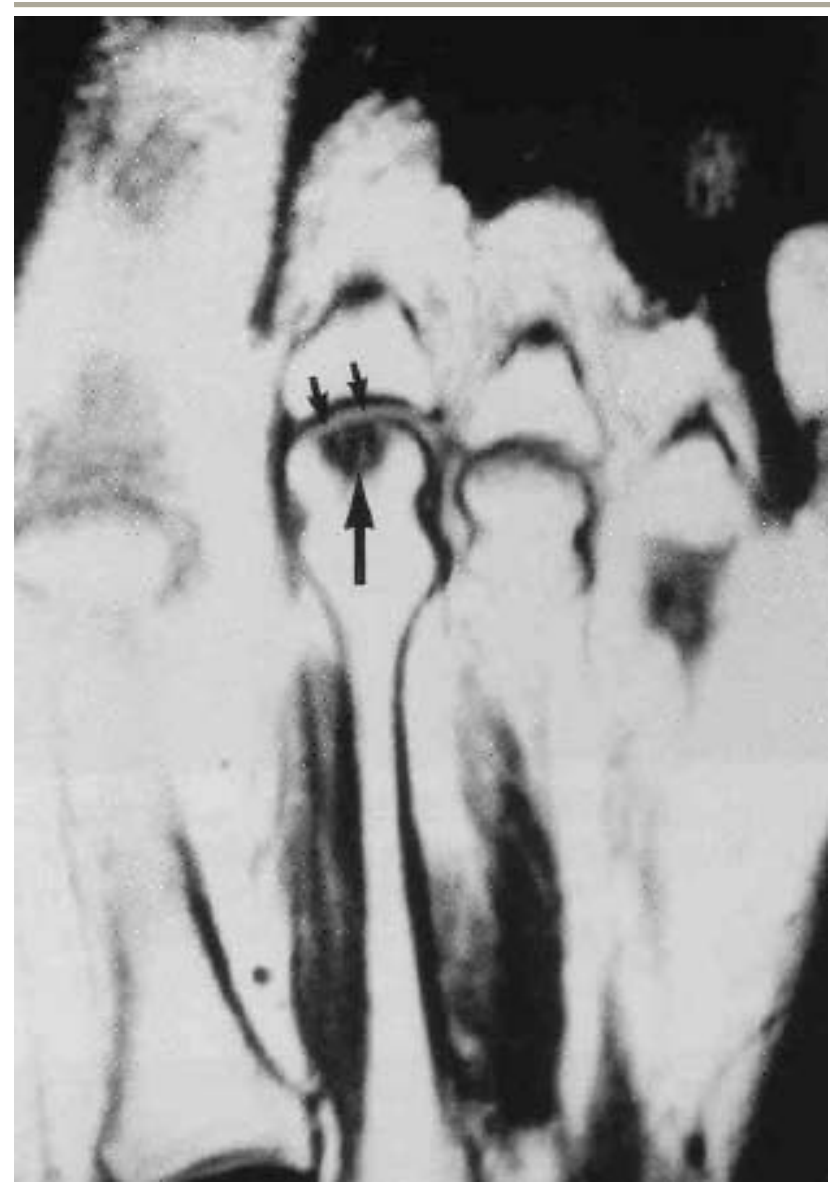

Figure 1 Early flattening (small arrows) with low signal intensity osteochondrosis of the second metatarsal head on a T-1 axial image. (Courtesy David W. Stoller, MD: Magnetic Resonance Imaging in Orthopaedics \& Sports Medicine, pp.488-489, 1993.)

Patients usually present with a painful and often swollen joint. A history of trauma may or may not exist. There is usually point tenderness to the dorsal aspect of the second metatarsophalangeal joint and associated limitation of joint motion. Diagnosis is often confirmed by simple radiographs which show varying stages of metatarsal head injury and articular depression.

MR (magnetic resonance) imaging has been definitively shown to aid in the diagnosis of Freiberg's infraction before joint changes occur on radiograph. ${ }^{4}$ This may be particularly useful in the early stage of the disorder when joint pain is present without observed changes to the joint surface.

(C) The Foot \& Ankle Journal, 2008

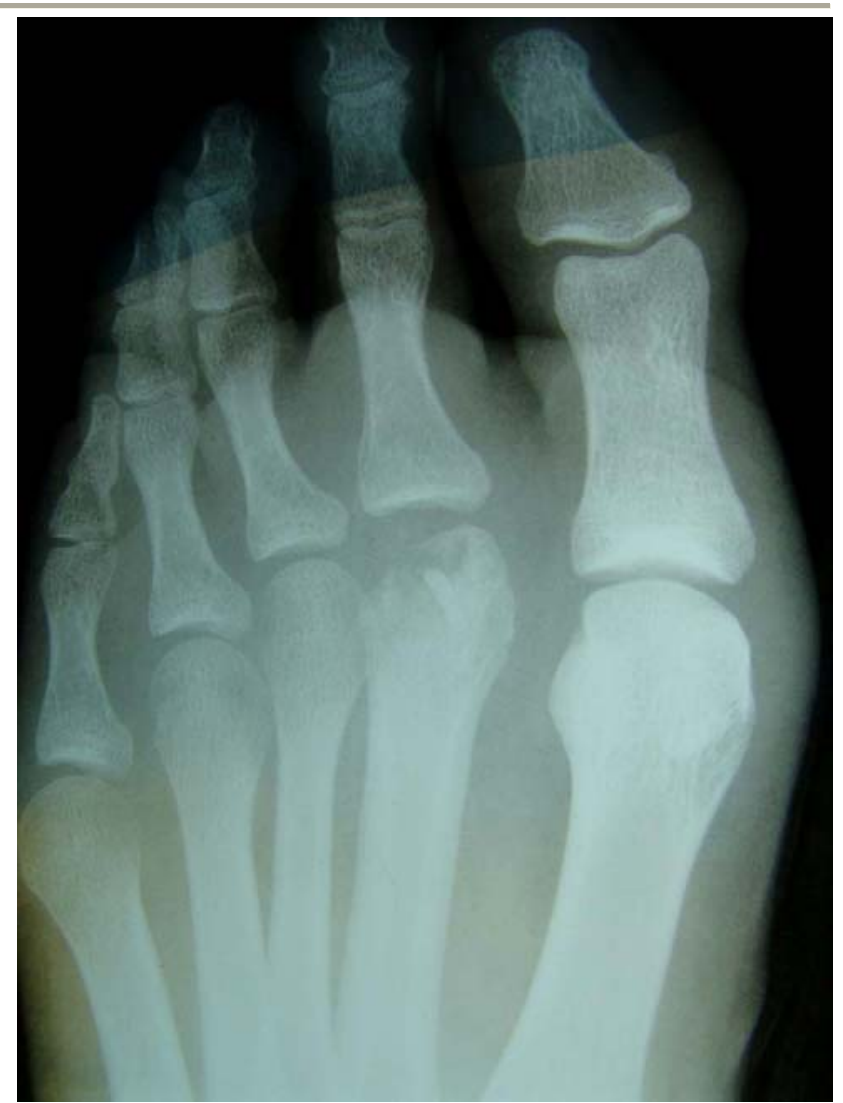

Figure 2 Initial radiograph shows subchondral disruption of the articular surface of cartilage involving the second metatarsal head. Note the long second metatarsal and Morton's toe.

In the patient with a normal or even wide joint space, MR imaging allows for early identification of Freiberg's infraction through low signal intensity changes of subchondral sclerosis. (Fig. 1)

In his original article, Freiberg described his treatment which included simple removal of the loose bodies in the joint. Historically, there have been a number of surgical approaches to the treatment of Freiberg's infraction. The use of bioorthologics is a relatively new approach to the treatment of osteochondral defects and injury. A case report is presented describing the use of the NEXA Osteocure ${ }^{\mathrm{TM}}$ bone graft for repair of a stage II Freiberg's infraction of the second metatarsal head. 


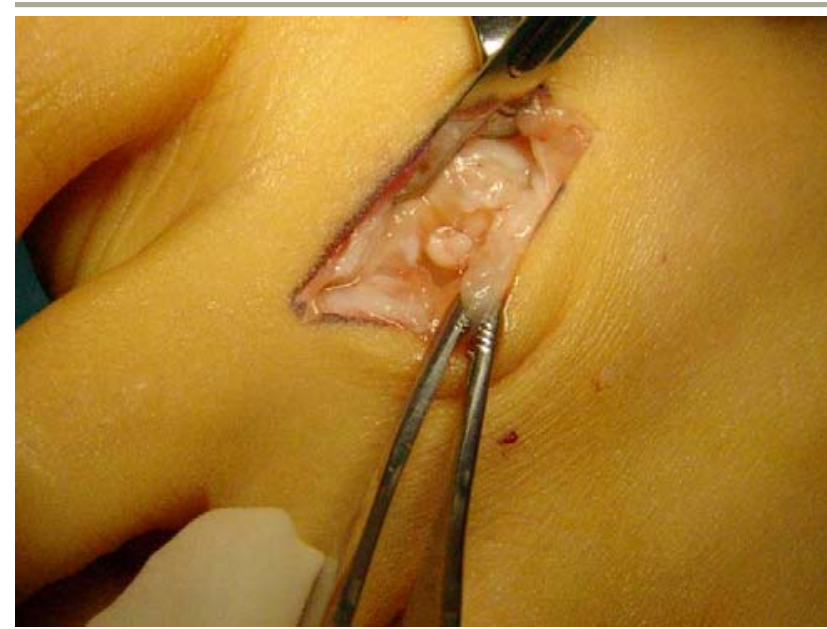

Figure 3 Once the joint is entered surgically, a dorsal linear capsulotomy reveals a loose joint body.

\section{Case Report}

An active fifteen year old female runner began having pain and discomfort to the second MTP joint. She did not recall any injury. She is very active in track and basketball. She has been running daily and relates to pain while running. She was using a silicone sleeve over the second toe and takes Tylenol and Motrin for pain and swelling. Her pain began to interfere with her running and she began seeking medical attention.

Her clinical examination revealed a swollen second metatarsophalangeal joint with pain upon dorsiflexion of the second toe. Joint range of motion was limited to about 5 to 10 degrees of dorsiflexion with extreme pain. Radiographic evaluation confirmed second metatarsal head changes consistent with Freiberg's infraction and a long second ray with Morton's toe. (Fig. 2) She underwent MR imaging which revealed diffuse increased signal intensity with local tissue edema and hemorrhage. There was joint effusion with fragmentation of the metatarsal head consistent with Freiberg's infraction, stage II.

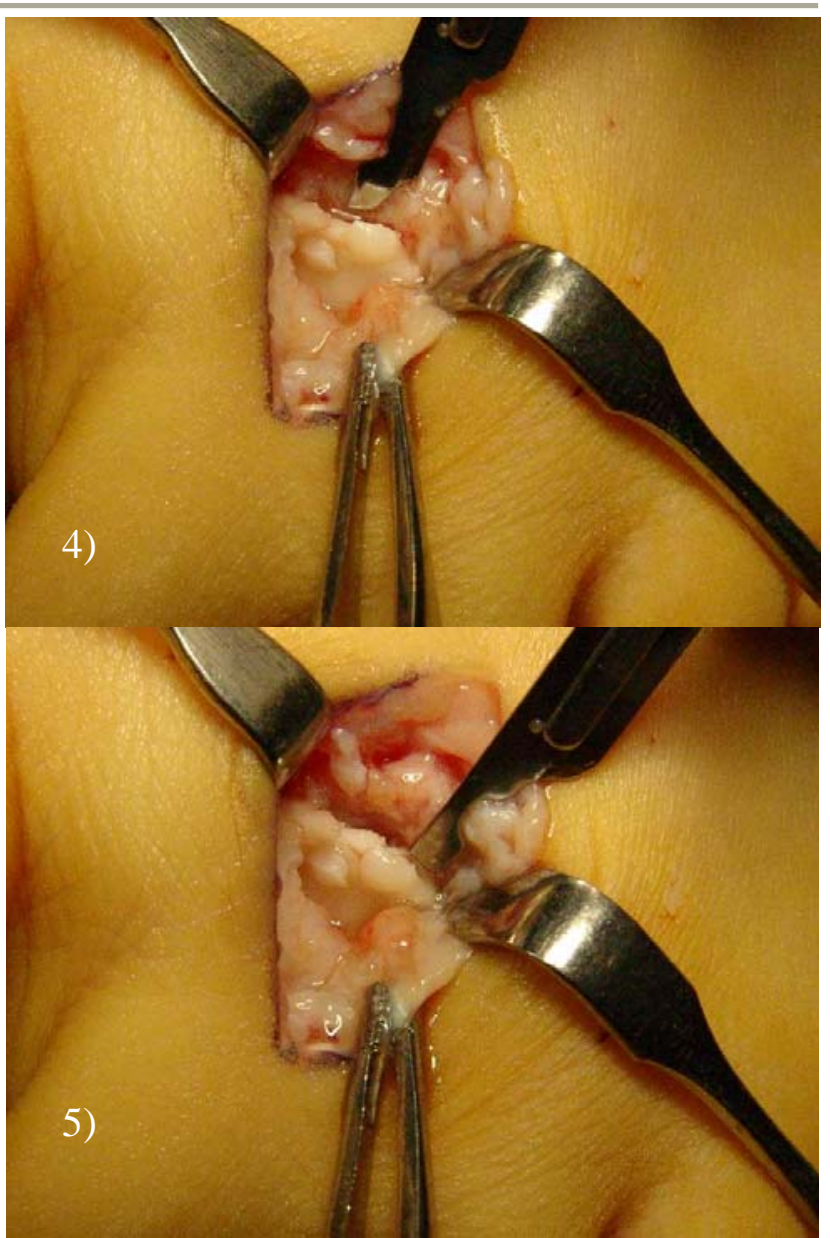

Figures 4 and 5 The loose cartilage is identified and demarcated from the subchondral bone. Care is taken to identify the plantar cartilaginous attachment.

The patient had a track meet within three weeks of our visit and returned about four weeks later and underwent casting and immobilization. After cast immobilization, she was placed in accommodative metatarsal padding and shoes and was asked not to return to running for an additional month. However, she continued to redevelop pain and swelling to the joint and was scheduled for surgery to include osteochondral bone repair with bioorthologic graft using the NEXA Osteocure ${ }^{\mathrm{TM}}$ bone graft. 


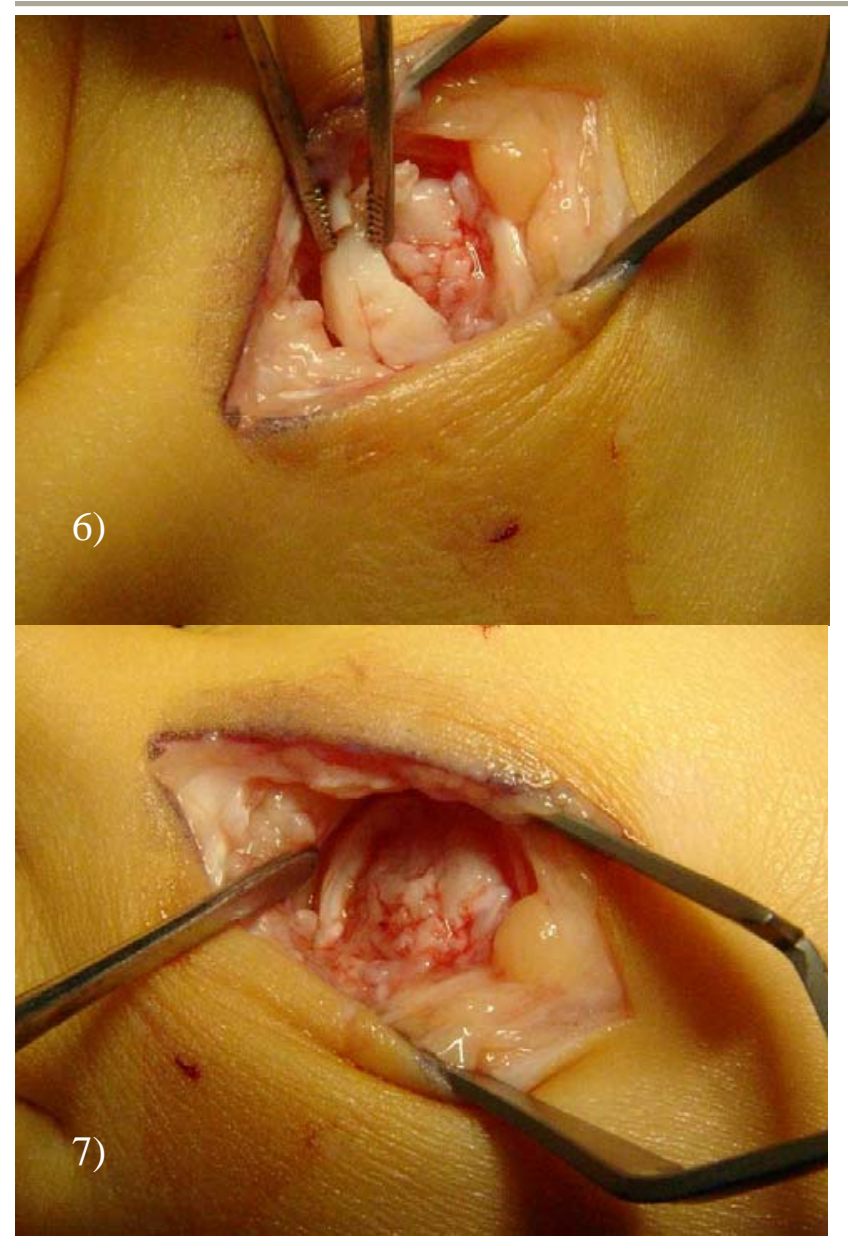

Figures 6 and 7 The loose cartilage is removed and the plantar cartilaginous attachment is identified. A semi-circular excision of the cartilage flap has been performed.

\section{Surgical Technique}

The patient was brought to surgery. A local second ray block was performed under intravenous sedation. A small incision was made directly over the second MTP joint. A dorsal capsular incision was performed and upon entry of the joint, we noticed a cartilaginous loose body at the dorsal aspect of the metatarsal head. (Fig. 3) The soft tissue capsule is delicately reflected to expose the cartilaginous surface of the metatarsal head.

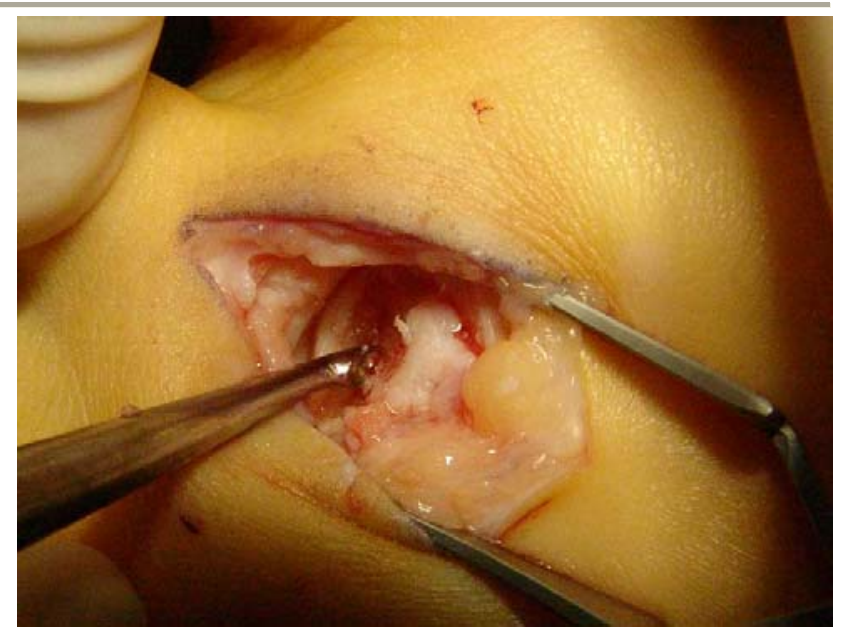

Figure 8 A curette is used to remove any diseased subchondral bone. The diseased portion of bone is usually softer than the underlying cancellous bone.

A large defect along the dorsal half of the metatarsal head is identified. The cartilage was actually separated from the underlying subchondral bone.

Using a small scalpel, the defect is lifted from the subchondral bone. It is important to identify the loose margin of cartilage and the point of firm attachment of the cartilage cap plantarly in order to preserve the plantar cartilage. (Figs. 4 and 5) Once this margin is identified, the loose cartilage is then excised in a semi-circular fashion. This is done to facilitate the bone plug, which is round in its shape. (Figs. 6 and 7)

A curette is then used to remove the soft, unhealthy subchondral bone. (Fig. 8) Preparation of the site is probably the most crucial aspect of this surgery. In particular, joint range of motion needs to be unimpeded dorsally and requires the removal of joint spurring, loose bodies and bone defects. Using the NEXA Osteocure ${ }^{\mathrm{TM}}$ Implant Kit, a corresponding set of color-coded sizers allows for determination of the bone graft size. 


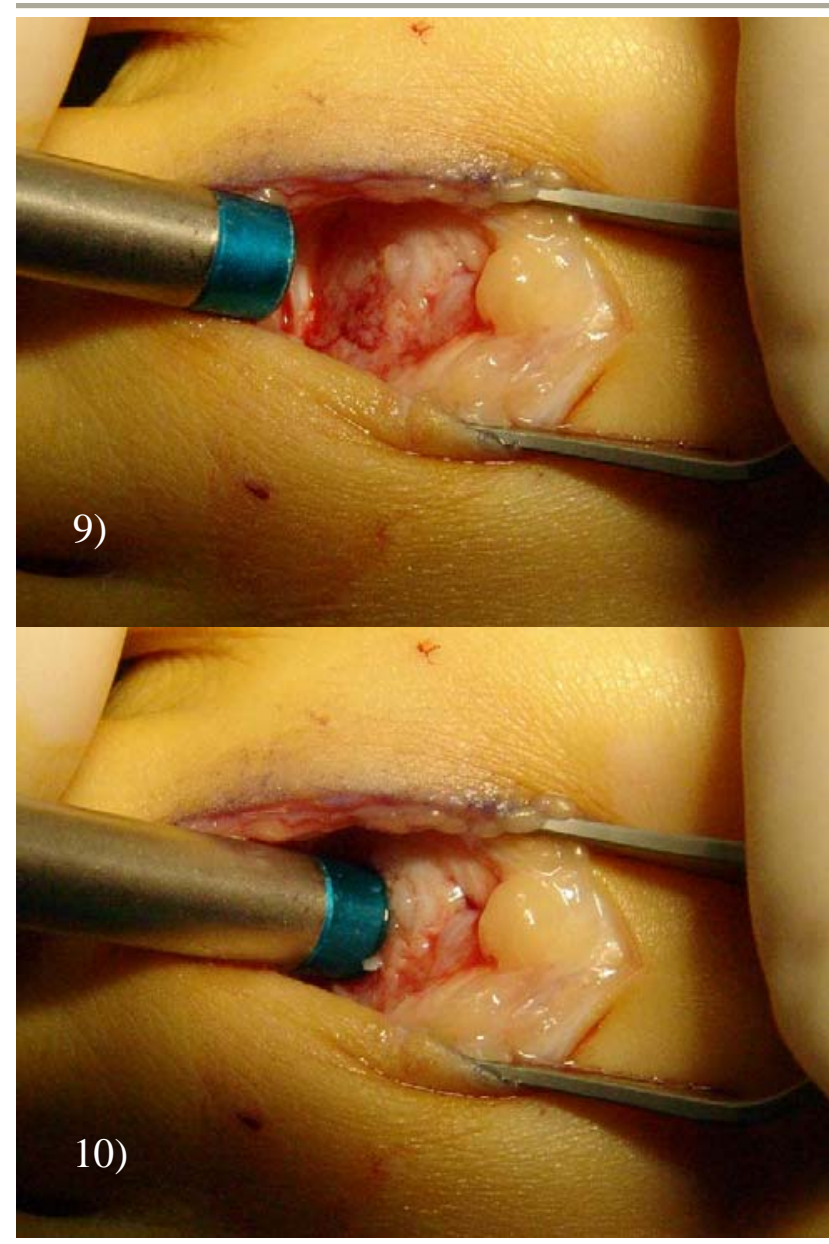

Figures 9 and $\mathbf{1 0}$ The bone plug sizer is used to determine the size diameter of the graft. Again, the inferior, semi-circular portion of healthy cartilage should fit directly to the sizer.

Color coded sizers of 5, 7, 9 and $11 \mathrm{~mm}$ diameter correspond to the respective graft size plugs. (Figs. 9,10) Once the graft size is determined, a reaming drill is used to prepare the graft site for implantation. (Figs. 11,12 and 13) The graft is then measured for depth, inserted and tapped within the newly prepared host site. (Figs. 14,15 and 16) The graft is then firmly placed into the host site. The joint can also be placed through its proper range of motion. This will ensure that the graft is articulating with the proximal phalanx. (Figs. 17 and 18)

The surgical site is then closed in the customary manner. The patient was then placed non-weightbearing in a posterior splint and kept on crutches for 4 weeks.

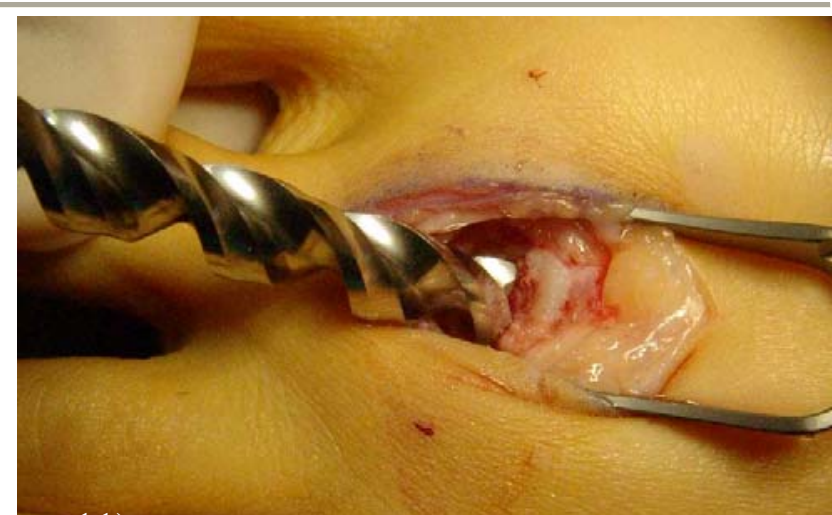

11)

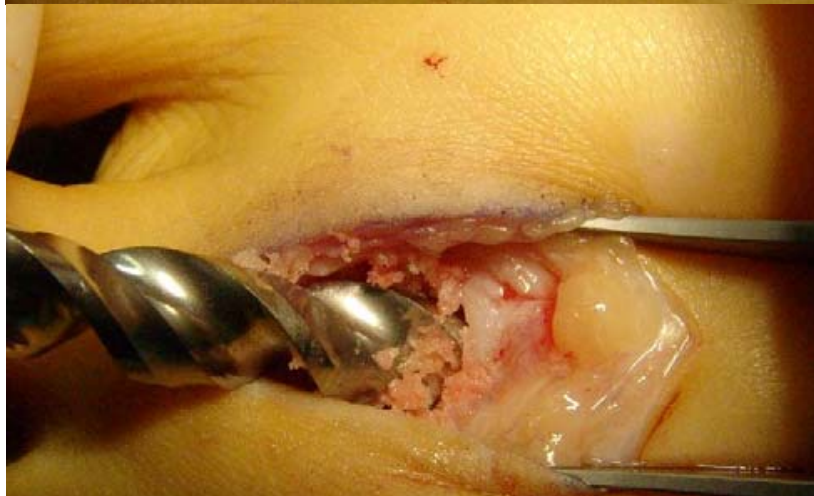

12)

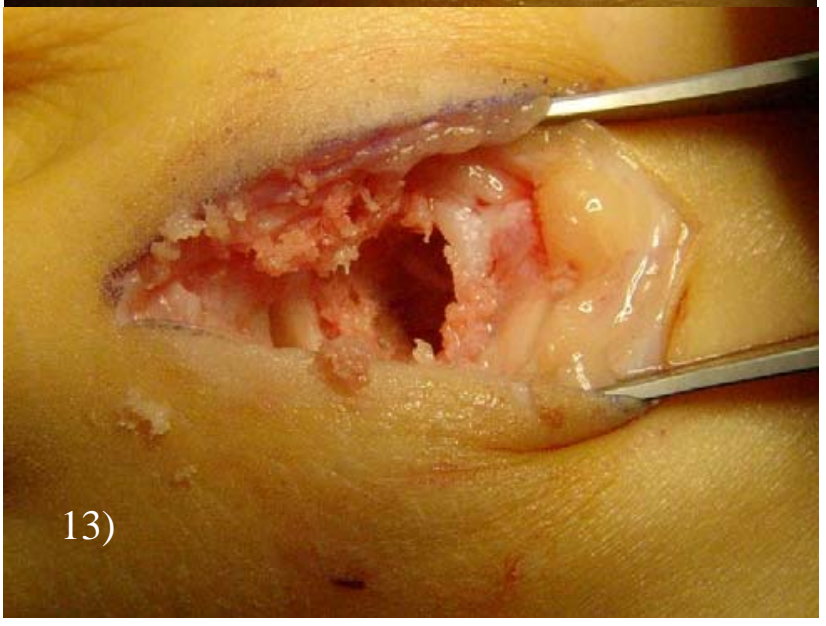

Figures 11,12 and 13 The drill corresponding to the sizer is used to prepare the host site for the graft plug.

This was followed by 2 weeks of partial weightbearing in a walking shoe. She was able to wear her athletic shoes within 8 weeks after surgery.

(C) The Foot \& Ankle Journal, 2008 


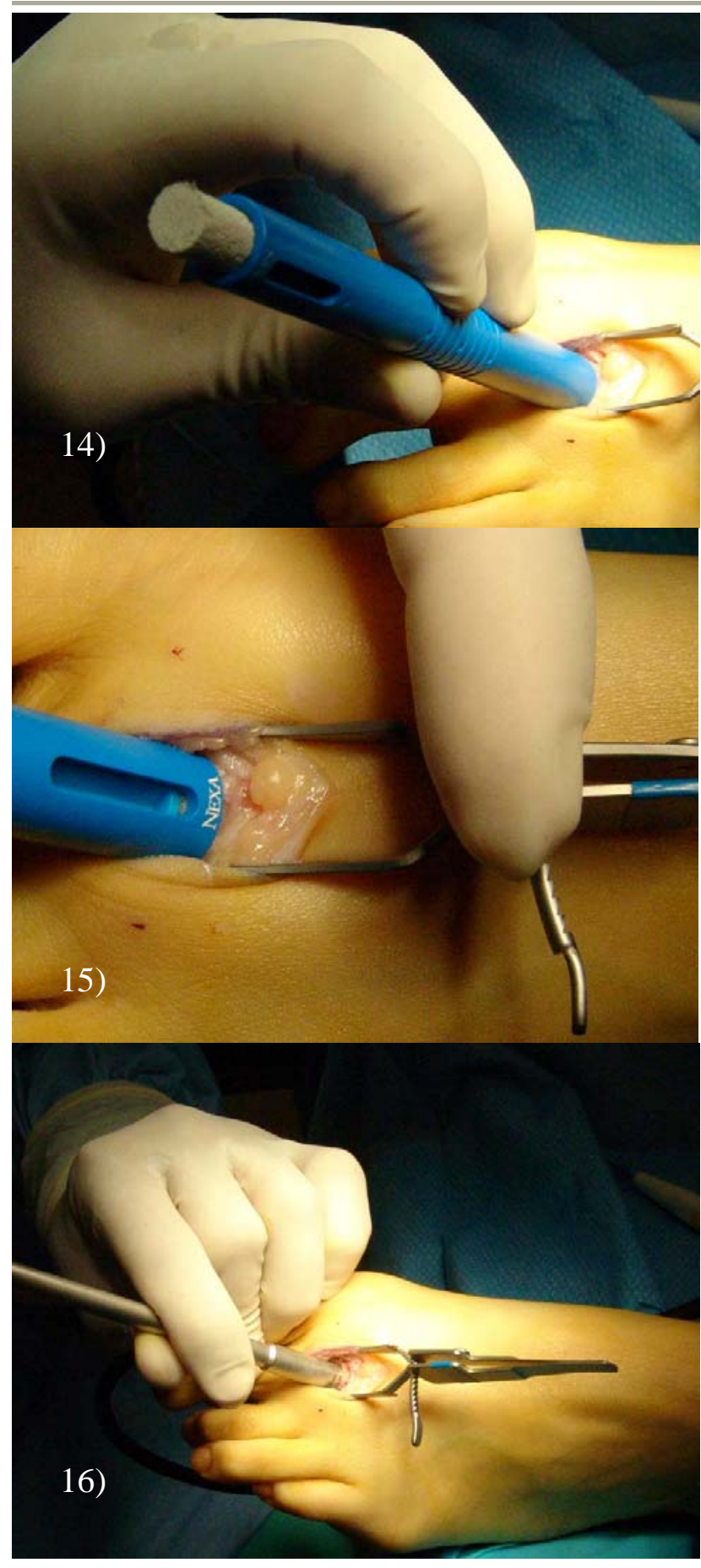

Figures 14,15 and 16 The graft is measured for depth and then inserted into the host site. A small tap is used to secure the graft into the host site.
The patient was pain free with an increase in joint range of motion. At last visit, she was playing volleyball without pain. (Figs. 19 and 20), however, the patient has been advised not run for six months after surgery to allow the graft to resorb.

\section{Discussion}

Several synonyms have been used to described Freiberg's infraction including Freiberg's infarction, osteochondrosis of the second metatarsal head, eggshell fracture, Koehler second disease, peculiar metatarsal disease, Panner disease of the metatarsal, osteochondritis deformans metatarsojuvenilis, malacopathia, subchondral bone fatigue fracture of the second metatarsal head and dorsal fatigue stress injury of the second metatarsal head. 2,7

In the majority of cases, the defect is usually dorsally located to the upper half of the articular surface of the metatarsal head. This would also lend support to the etiology of trauma causing this defect. In observing a number of these cases, mechanical changes appear to cause a dorsal articular jamming and trauma to the joint surface. These include a Morton's toe or long second digit, a short first metatarsal or elongated second ray and instability of the first ray. Limitation of dorsiflexory motion of the second MTP joint will lead to progressive joint damage and eventual articular shear. This will cause an actual lifting and separation of the articular cartilage from the subchondral bone disrupting the vascular supply to the articular cartilage.

Once the articular cartilage separates, the cartilage can fragment. The fragments that separate from the articular cartilage are called joint bodies or joint 'mice'.

As previously stated, 'infraction' is sometimes called Freiberg's 'infarction'. Infarction would suggest a vascular event leading to osteonecrosis. It has been suggested that avascular necrosis from injury will lead to the growth plate or epiphyseal injury in young, growing bone. This may also explain why the incidence is higher in young women and girls. 


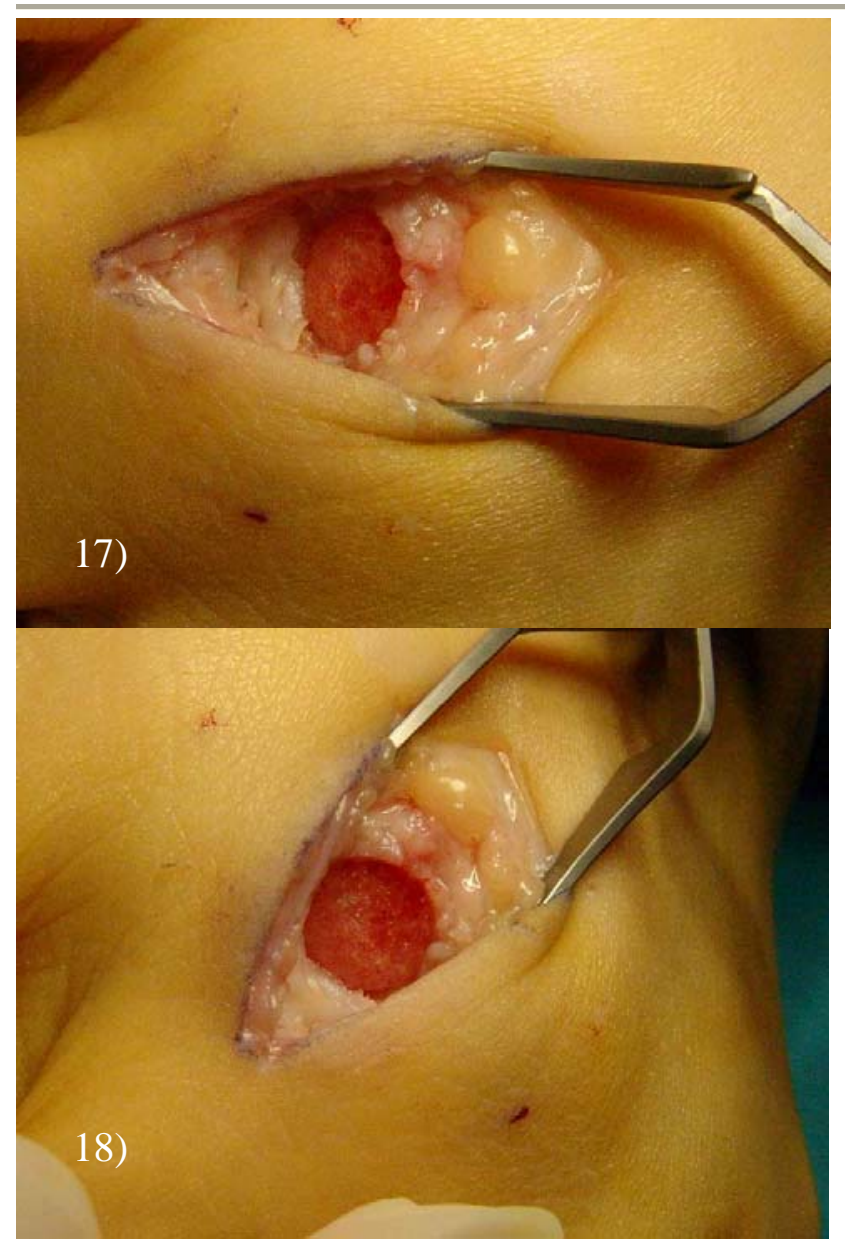

Figures 17 and 18 The graft is seated in the defect. The Osteocure ${ }^{\text {TM }}$ graft provides a press fit to the defect with close approximation to the surrounding bone and tissue causing migration of tissue into the scaffolding. This surface will provide scaffolding for hyaline-like cartilage in 6 to 12 months.

In 1959, Braddock observed that there is a relative weakness of the second metatarsal epiphysis at a certain stage of epiphyseal maturation. ${ }^{3}$ Whatever the etiology, the result appears to cause cartilage lifting and separation from the underlying subchondral bone and vascular osteonecrosis of the joint surface.

The extent of articular damage however, appears to be directly proportional to the extent of joint pain.

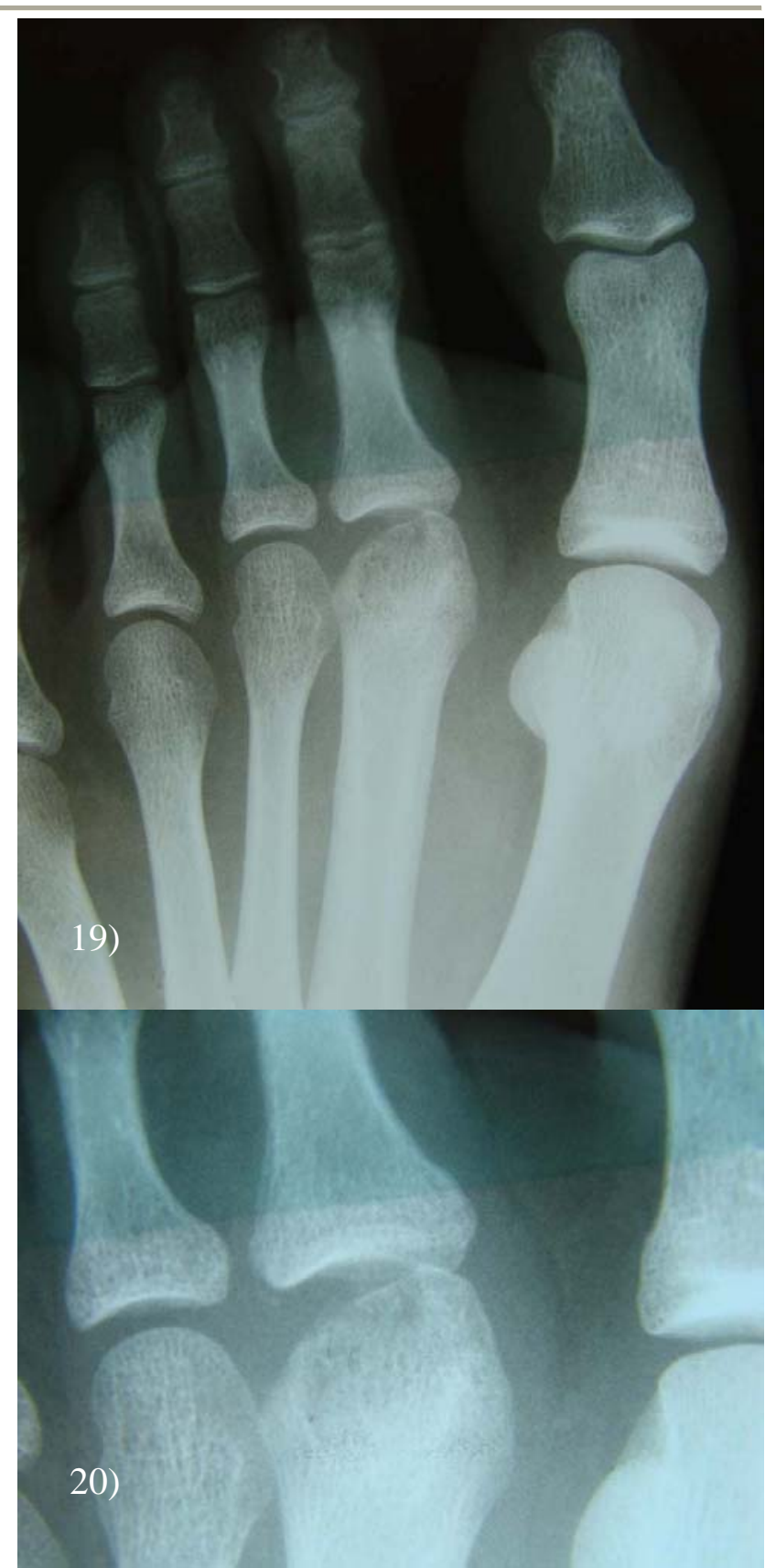

Figures 19 and 20 Two months following surgery, incorportation of the graft is already seen. A thin articular margin is also appreciated. Although, there is still some flattening of the metatarsal head, the patient is now asymptomatic. A close up of the radiograph (Fig. 20) reveals new subchondral bone formation in a cancellous pattern with metatarsal head resurfacing. 
Treatment, including both conservative and surgical approaches, varies to the extent of injury. In early stages, where the articular cartilage may form a small depression, treatment usually consists of immobilizing the joint.

Conservative treatments should almost always consist of immobilization, offloading and casting irrespective of severity and stage. The goal of non-operative immobilization is to promote a decrease in joint effusion and inflammation including the surrounding soft tissue structures. This may be supplemented with NSAID therapy, joint injections and oral steroids. In patient's who may not want surgery, shoe modification, metatarsal bars, orthotics and even rocker bottom shoes may provide some temporary relief. In a majority of cases, operative intervention is recommended to provide improved joint mechanics, remove damaged joint spurs and loose joint bodies and treat the osteochondral defect.

\section{Methods of Surgical Treatment}

Freiberg initially described removal of the loose bodies to the affected joint. Other treatments have been more aggressive, including the actual removal of the metatarsal head. Unfortunately, this will lead to more complicated conditions of the foot including transfer metatarsalgia, stress fracture and contracture of the corresponding digit.

Some authors have also suggested joint arthroplasty and resecting the base of the proximal phalanx. ${ }^{6}$ This may decompress the joint, but joint instability and toe shortening or dorsiflexory rotation of the digit is a major complication.

In 1991, Smith, et al., described simple decompression osteotomy using a T-plate in 15 patients with Freiberg's infraction of the second metatarsal head. They reported good results and relief of pain within 12 months, although all patients had limited motion of the joint postoperatively. $^{5}$

(C) The Foot \& Ankle Journal, 2008
Dorsiflexory osteotomies have also been described in order to rotate the healthy plantar cartilage dorsally. This is commonly performed by a v-osteotomy and use of cross K-wire technique or screw fixation. However, rotational osteotomies are only indicated in Freiberg's stages I-III where the plantar hinge of the cartilage is still intact. Implants have also been used including silastic and titanium total implants and titanium hemi-implants. Shih, et al., described the successful use of a titanium hemi-implant in the treatment of Freiberg's infraction. They reported a successful increase in range of motion of the joint from 15 degrees to 45 degrees, with no pain or activity limitation. ${ }^{6}$

\section{The advantage of using bioorthologics in the treatment of osteochondral defects}

Newer techniques are now being introduced in an attempt to actually repair the damaged cartilage and subchondral bone in Freiberg's infraction. Orthobiologic materials, particularly PolyGraft ${ }^{\mathrm{TM}}$ materials, provide a scaffolding for regeneration of tissue and have demonstrated evidence of resurfacing the articular cartilage with hyaline-like cartilage. This is different than resurfacing of the joint with fibrocartilage after damage. Fibrocartilage is primarily Type I collagen and is less desirable than Hyaline cartilage, which is primarily comprised of type II collagen. In a recent land-mark study, Cascio, et al., reported exciting chondral repair with observed hyaline cartilage in a goat model using an injectable hydrogel bone graft. ${ }^{8}$

Critical size chondral defects were created on the stifle joint of adult goats. Experimental defects underwent marrow stimulation to recruit reparative stem cells and were primed with a chondroitin-sulfate based adhesive to anchor the scaffold. Controls received marrow stimulation alone. 
The operative goat legs were cast for two weeks and after 13 weeks, cartilage fill and repair quality was assessed by histomorphometry evaluation. Results revealed evidence of hyaline-like repair with a $12 \%$ increase in total tissue fill in scaffoldtreated defects on histomorphometry. Remodeling and articular cartilage bridging was seen up to twelve months after implantation.

This is an exciting development in the treatment of osteochondral defects by actually healing the cartilage defect with desirable hyaline cartilage and will maintain the joints normal cartilaginous properties. The Osteocure ${ }^{\mathrm{TM}}$ bone plug is a porous PolyGraft ${ }^{\mathrm{TM}}$ and is the first $100 \%$ resorbable composite scaffold material designed to fill osteochondral defects. Its properties include a three dimensional scaffolding structure for tissue incorporation and remodeling. Resorbable polymers and bioactive ceramics are combined to produce the desired strength, stiffness and bone growth characteristics. It also withstands mechanical loads and compression without crushing the material. The Osteocure ${ }^{\mathrm{TM}}$ graft provides a press fit to the defect with close approximation to the surrounding bone and tissue causing migration of tissue into the scaffolding. The hydrophilic properties of the porous scaffolding allow blood, marrow and progenitor cells to wick into the pores and provide a stable environment for tissue in-growth and cellular development. Between six and twelve months, the polymer graft is resorbed and replaced with living tissue. ${ }^{9}$

\section{Conclusion}

Although there is no one consensus on the best surgical treatment for this condition, the use of orthobiologics provides a promising new attempt to restoring the cartilaginous surface of the joint and eliminating joint destruction and pain. More importantly, these products appear to heal and replace the natural hyaline cartilage of the joint surface.

\section{References}

1. Freiberg, AH. Infraction of the second metatarsal head, a typical injury. Surg Gyn Ob, (19) 163-191, 1914.

2. Boyer, M., DeOrio, J. Freiberg Infraction. E-Medicine, Online article, 2004.

3. Braddock GTF. Experimental epiphyseal injury and Freiberg's disease. JBJS, 41B (1) February, 1959.

4. Stoller DW. Magnetic Resonance Imaging in Orthopaedics and Sports Medicine. J.B. Lippincott Company, Philadelphia, $1^{\text {st }}$ edition, 1993.

5. Smith TW, Stanley D, Rowley DI. Treatment of Freiberg's disease: A New Operative Technique. JBJS 73B (1), January, 1991.

6. Shih AT, Quint RE, Armstrong DG, Nixon BP. Treatment of Freiberg's infraction with the Titanium hemiimplant. JAPMA 94 (6), 590-593, Nov/Dec 2004.

7. Blitz NM, Yu JH. Freiberg's infraction in identical twins: A case report. JFAS, 44(3), May/June 2005.

8. Cascio B, Sharma, MS, Fermanian, BS, Elisseeff, J: Chondral lesion repair in a critical size defect model using injectable hydrogel scaffold in conjunction with marrow stimulation. AOSSM, Poster \#32, 2006.

9. Nexa Orthopaedics: Osteocure: PolyGraft ${ }^{\mathrm{TM}}$ Material Technology. [Online PDF] 